Supporting Information

\title{
Three-dimensional Photonic Crystal Bulks with Outstanding Mechanical Performance Assembled by Thermoforming-Etching Cross- linked Polymer Microspheres
}

Yue Fu, Yunming Wang, * Dan Chen, Zhaohan Yu, Jiaqi Zheng, Huamin Zhou

State Key Laboratory of Material Processing and Die \& Mould Technology, School

of Materials Science and Engineering, Huazhong University of Science and

Technology, Wuhan 430074, China.

*Email: wang653@hust.edu.cn. Phone: +86 027-87543492. 
To research the optimal dosage of the crosslinking agent (Divinylbenzene, DVB), we synthesized $220 \mathrm{~nm}$ cross-linked PS microspheres with five different DVB concentrations: $1 \mathrm{wt} \%, 3 \mathrm{wt} \%, 5 \mathrm{wt} \%, 7 \mathrm{wt} \%$ and $10 \mathrm{wt} \%$. To realize blue and red structural color, we synthesized 7 wt \% DVB cross-linked PS microspheres with the mean microsphere diameters of $180 \mathrm{~nm}$ and $265 \mathrm{~nm}$. The SEM images of the microspheres show good monodispersity (Figure S1a and b). Thermogravimetric analysis (TGA) was conducted to characterize the thermal stability of $220 \mathrm{~nm}$ crosslinked PS microspheres (1 wt \%, 3 wt \%, 5 wt \%, 7 wt \% and 10 wt \% DVB) (Figure S1c), illustrating that the DVB concentration had little effect on the thermal stability of microspheres. A significant drop in weight occurred in the microspheres during the temperature interval of $360-410^{\circ} \mathrm{C}$, which caused by decomposition of polymer chains. The differential scanning calorimetry (DSC) curve (Figure S1d) illustrates that the $\mathrm{T}_{\mathrm{g}}$ (glass transition temperature) of the cross-linked PS microspheres is approximately $120-130{ }^{\circ} \mathrm{C}$. The viscosity curves (Figure S1e) show good fluidity of cross-linked PS microspheres (7 wt \% DVB; $220 \mathrm{~nm})$ at $170{ }^{\circ} \mathrm{C}$. 

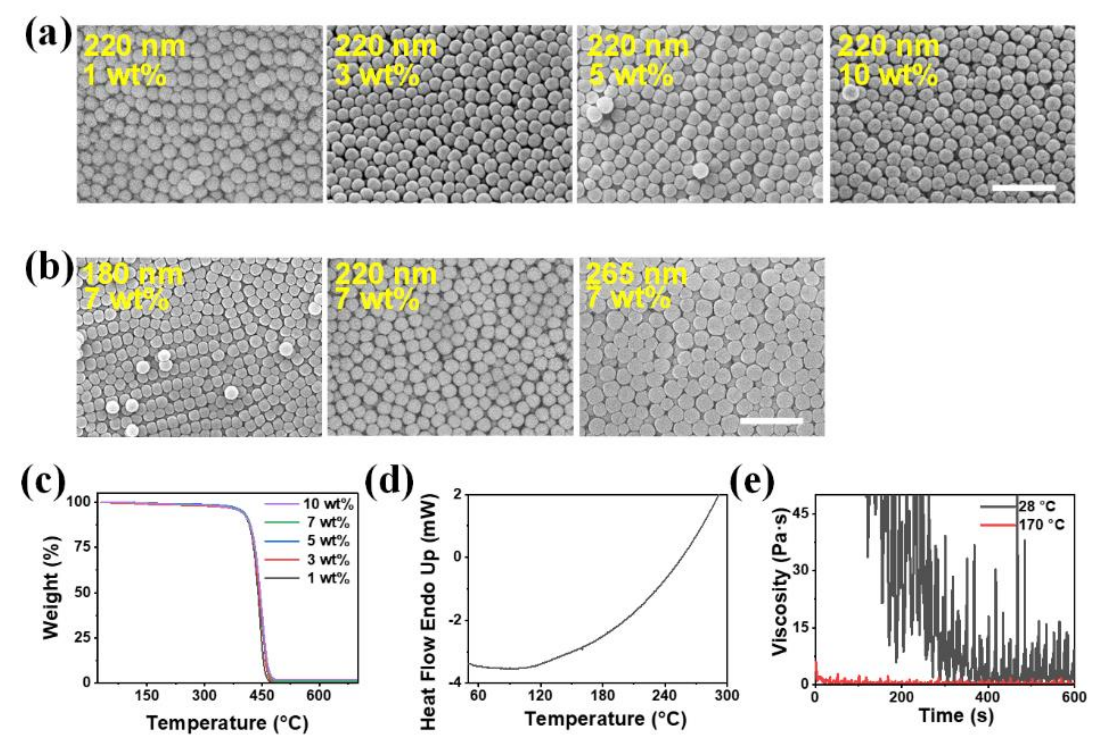

Figure S1. (a) SEM images of the $220 \mathrm{~nm}$ PS microspheres powder with $1 \mathrm{wt} \%, 3$ wt \%, $5 \mathrm{wt} \%$ and $10 \mathrm{wt} \%$ DVB; (b) SEM images of the $7 \mathrm{wt} \%$ DVB PS microspheres powder with particle diameters of $180 \mathrm{~nm}, 220 \mathrm{~nm}$ and $265 \mathrm{~nm}$; (c) TGA curves of the $220 \mathrm{~nm}$ cross-linked PS microspheres with different DVB concentrations; (d) DSC curve of the cross-linked PS microspheres (7 wt \%; $220 \mathrm{~nm}$ ); (e) The fluidity curves of the PS microspheres powder (7 wt \%; $220 \mathrm{~nm})$. Scale bar: (a) and (b) $1 \mu \mathrm{m}$.

The bulk PC is approximately modelled as a three-dimensional photonic crystal with face-centered cubic structure. The reflected wavelength at maximum reflectivity of the PCs were described as follows, ${ }^{1-3}$

$$
\begin{aligned}
& \lambda_{\max }=1.633 d\left(n_{\text {eff }}{ }^{2}-\sin ^{2} \theta\right)^{1 / 2} \\
& n_{\text {eff }}{ }^{2}=0.74 n_{\text {sphere }}{ }^{2}+0.26 n_{\text {air }}{ }^{2}
\end{aligned}
$$

where $\lambda_{\max }$ is the reflected wavelength at maximum reflectivity, $d$ is the mean microsphere diameter of the bulk PC, $n_{\text {eff }}$ is the effectively refractive index, $\theta$ is the incident angle, $n_{\text {sphere }}$ and $n_{\text {air }}$ are the refractive index of the microsphere and air, respectively. The 0.74 and 0.26 are the space of PS microspheres and the space of air gap, respectively. 
According to the Bragg law, ${ }^{1-3}$ the ratios of the theoretical reflected wavelength at maximum reflectivity to the actual were calculated in Table S1. The ratios are approximately 0.93 at $10^{\circ}$ angle, illustrating the microstructure of the bulk PCs is similar to complete PC structure.

Table S1. Ratios of the theoretical reflected wavelength of the bulk PCs at maximum reflectivity to the actual.

\begin{tabular}{ccccc}
\hline$\theta$ & $d$ & $\lambda_{\text {theoretical }}$ & $\lambda_{\text {actual }}$ & Ratio of $\lambda_{\text {theoretical }}$ to $\lambda_{\text {actual }}$ \\
\hline \multirow{3}{*}{$10^{\circ}$} & $180 \mathrm{~nm}$ & $426 \mathrm{~nm}$ & $456 \mathrm{~nm}$ & 0.93 \\
& $220 \mathrm{~nm}$ & $521 \mathrm{~nm}$ & $551 \mathrm{~nm}$ & 0.94 \\
& $265 \mathrm{~nm}$ & $627 \mathrm{~nm}$ & $672 \mathrm{~nm}$ & 0.93 \\
\hline $70^{\circ}$ & $180 \mathrm{~nm}$ & $328 \mathrm{~nm}$ & $381 \mathrm{~nm}$ & 0.86 \\
& $220 \mathrm{~nm}$ & $401 \mathrm{~nm}$ & $453 \mathrm{~nm}$ & 0.88 \\
\hline
\end{tabular}

As shown in Figure S2a, the cross-linked PS microsphere powder was fabricated to PS bulk after thermoforming, and then the bulk PC $(180 \mathrm{~nm})$ with blue structural color was obtained after etching the PS bulk. The thermoforming conditions were $200{ }^{\circ} \mathrm{C}$ at $220 \mathrm{MPa}$ and kept pressure for $30 \mathrm{~min}$. The diameter and thickness of the mold gasket made of die steel are $30 \mathrm{~mm}$ and $10 \mathrm{~mm}$, respectively. Soaking the PS bulk in a mixed solvent $\left(\mathrm{V}_{\text {Ethyl Acetate }} / \mathrm{V}_{\text {Anhydrous Ethanol }}=1: 1\right)$ for $25 \mathrm{~min}$ to realize etching process.

As shown by the reflectance spectrums (Figure S2b and c), the Bragg peak of the bulk PC assembled by $180 \mathrm{~nm}$ microspheres was higher and narrower than $265 \mathrm{~nm}$ microspheres due to the more ordered microstructure.

To specify the effect of microsphere size on the mechanical performance of the bulk PCs, nanoindentation analysis was performed (Figure S2d). The response curve of the bulk PCs (180 nm, $220 \mathrm{~nm}$ and $265 \mathrm{~nm})$ show that the modulus decreased slightly first and then increased slightly (the values are $4.13 \mathrm{GPa}, 3.95 \mathrm{GPa}$ and $4.12 \mathrm{GPa}$, 
respectively) and hardness increased slightly first and then decreased slightly with increasing microsphere size (the values are $0.13 \mathrm{GPa}, 0.17 \mathrm{GPa}$ and $0.13 \mathrm{GPa}$, respectively). Additionally, the mechanical performance of the bulk PC (220 nm) before and after etching were roughly the same which illustrated the mechanical strength of the bulk PC was formed during thermoforming process. Furthermore, the pressure sustained by a single microsphere was as high as $2.1 \times 10^{3} \mathrm{GPa}$; thus, the molten PS molecules flowed out from surface of the microspheres and were entangled with each other, and then, the continuous mechanical strength of the bulk PC was reached.

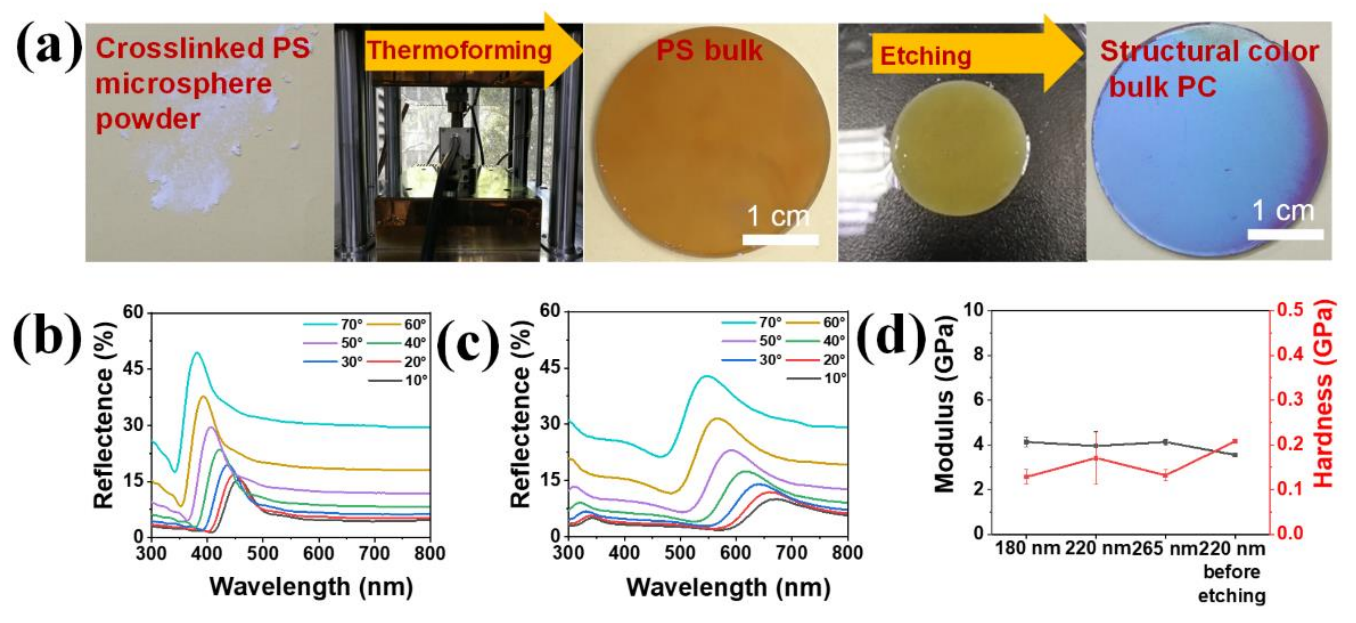

Figure S2. (a) Thermoforming-etching process of the $180 \mathrm{~nm}$ cross-linked PS microspheres; (b) The reflectance spectrum of the bulk PC (7 wt \%; $180 \mathrm{~nm}$ ); (c) The reflectance spectrum of the bulk PC (7 wt \%; $265 \mathrm{~nm})$; (d) The mechanical performance of the bulk PCs with different particle diameters.

Images of the PC bulks (220 nm) under different processing conditions are shown in Figure S3. The PS bulks with high DVB concentration at $130{ }^{\circ} \mathrm{C}$ thermoforming temperature showed a partially white area that illustrated partial microspheres could not transform to rubbery state at $130{ }^{\circ} \mathrm{C}$. The white area lacked of molten PS molecules, 
the microstructure was disordered and reflected white color after etching. With the increase of thermoforming temperature, PS bulk darkened which illustrated the decomposition of PS molecular chains under high pressure and temperature. PS microspheres with low DVB concentration were easier to decompose during thermoforming process and the thermal decomposition of PS microspheres increased with the increase of thermoforming temperature. The PS bulk in the lower-left corner became thinner and partial powder overflowed from the mold cavity, illustrating the fluidity of the microsphere powder increased with the increase of thermoforming temperature and the decrease of DVB concentration. To avoid all the microsphere powder overflowed from the mold cavity, the pressure and thermoforming temperature should be decreased during thermoforming process for microspheres with low DVB concentration. As the DVB concentration decreased and the thermoforming temperature increased, the gorgeous structural color could be achieved at a short etching time. (Table S2)

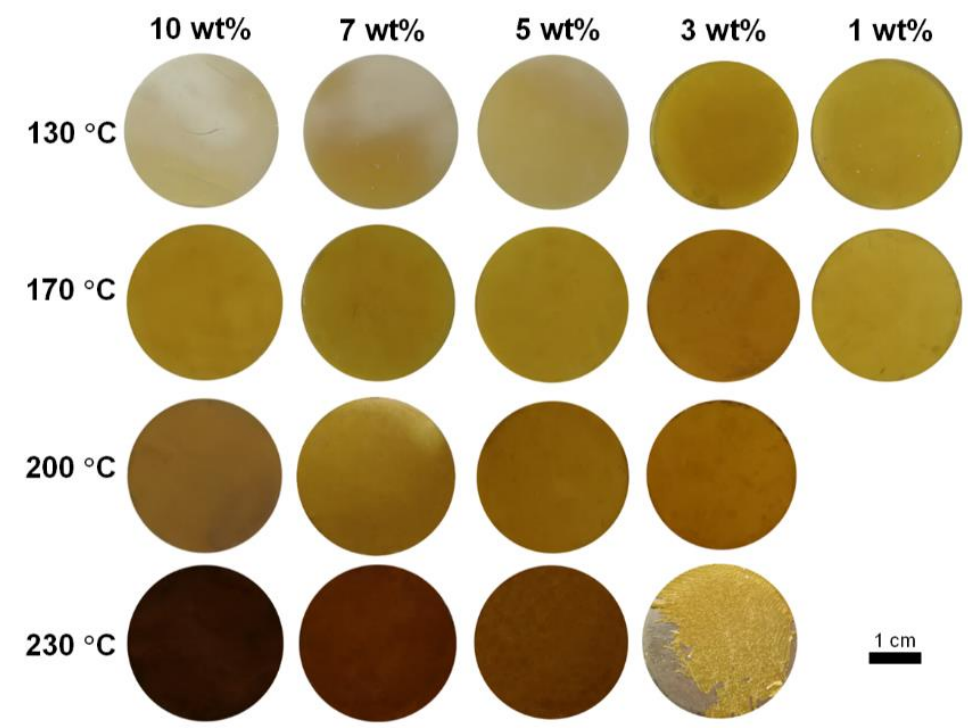

Figure S3. The images of PS bulks with different DVB concentrations processed under 
different thermoforming temperatures and processing conditions.

Table S2. The detailed processing conditions (DVB concentration, etching time, thermoforming pressure and temperature) of the bulk PCs corresponding to the PS bulks in Figure S3.

\begin{tabular}{cccccc}
\hline & & & \\
\end{tabular}

To obtain the optimum processing condition, structural, optical and mechanical performance of bulk PCs with different DVB concentrations processed at different thermoforming temperatures were characterized. The SEM images of the bulk PCs (Figure S4a) illustrated that the microstructure was disordered at $1 \mathrm{wt} \% \mathrm{DVB}$ and the microstructure defects were excessive at $10 \mathrm{wt} \% \mathrm{DVB}$, indicating that the molten PS molecules depended on the DVB concentration \& temperature had an important effect to the ordered microstructure. With the increase of thermoforming temperature, the microsphere gap size increased and surface flatness decreased indicating that molten PS molecules were increased and high swelling degree at high temperature.

The reflectance spectra of the bulk PCs (Figure S4b) illustrates that the reflectivity of the bulk PCs increased first and then decreased with the increase of the DVB concentration. With the increase of the temperature, the reflectivity was decreased, 
except for $7 \mathrm{wt} \%$ DVB which was increased first and then decreased. Comprehensively comparing the SEM images and reflectance spectra of the bulk PCs, we deduced the reflectivity is mainly affected by the microsphere gap size and order degree of bulk PCs, which are inextricably bound up with the DVB concentration and temperature. Under the optimal conditions $\left(200{ }^{\circ} \mathrm{C}\right.$; 7 wt \%), the bulk PC had a highly ordered microstructure and the max reflectivity was the highest.

The nanoindentation curves are roughly the same (Figure S4c), illustrating the similar mechanical performance of the bulk PCs under different conditions. The results illustrate thermoforming temperature had a little effect on modulus and hardness. The modulus and hardness values were roughly the same with increasing DVB concentration, except for the modulus values of the $5 \mathrm{wt} \%$ DVB bulk PCs which were lower than the others. 
(a)

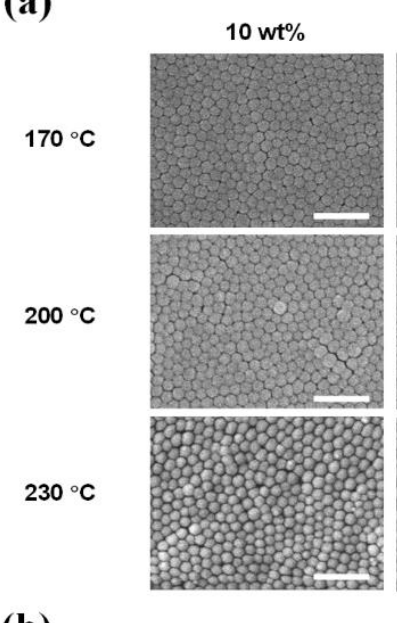

(b)
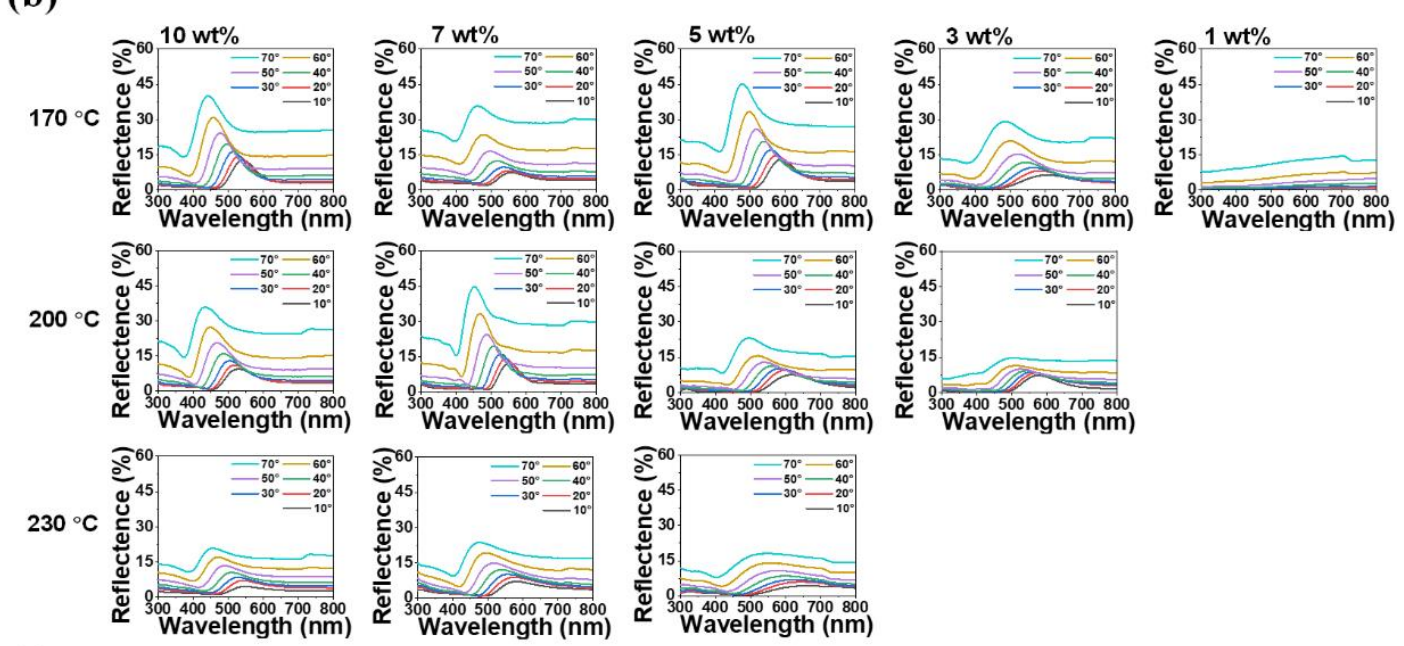

(c)
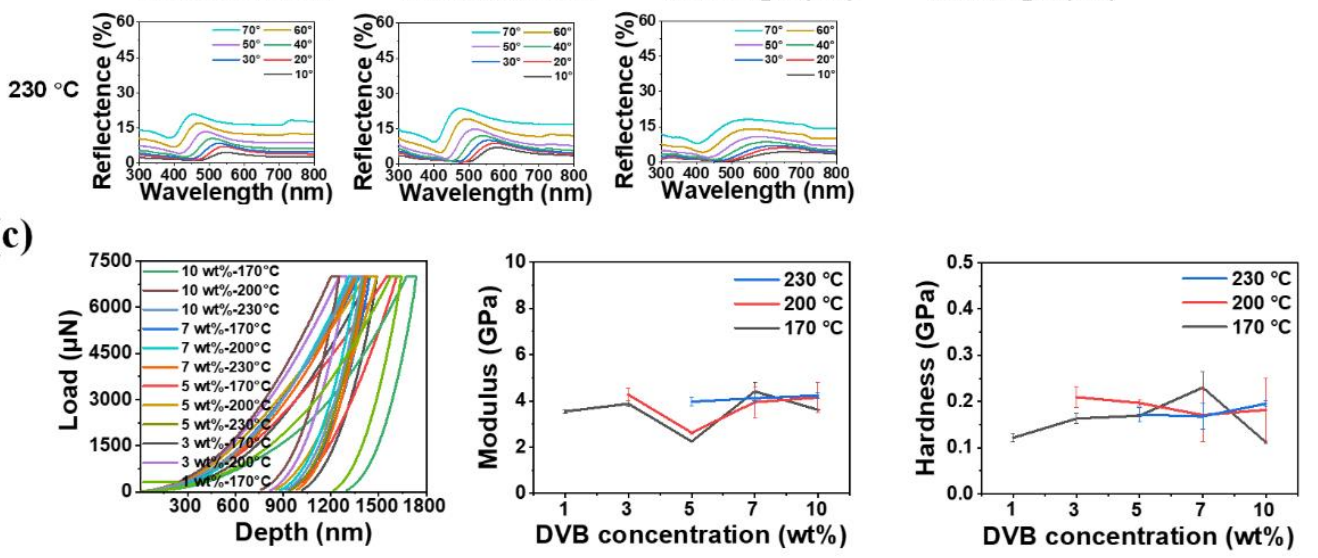

Figure S4. (a) SEM images of the bulk PCs after etching corresponding to the PS bulks before etching in Figure S3; (b) The reflectance spectrum of the bulk PCs corresponding to the SEM images in a; (c) Nanoindentation results of the bulk PCs corresponding to the SEM images in a. Scale bar: (a) $1 \mu \mathrm{m}$.

As shown in Figure S5a, to project and record the reflected color of bulk PC devices, white light was used to shine the devices at an angle. To print pattern on the devices, the PS bulk PCs pasted with hollow polyimide tape was soak in ethyl acetate for $2 \mathrm{~min}$ 
(Figure S5b). After the ethyl acetate volatilizing, gorgeous structural color pattern generated from the device.
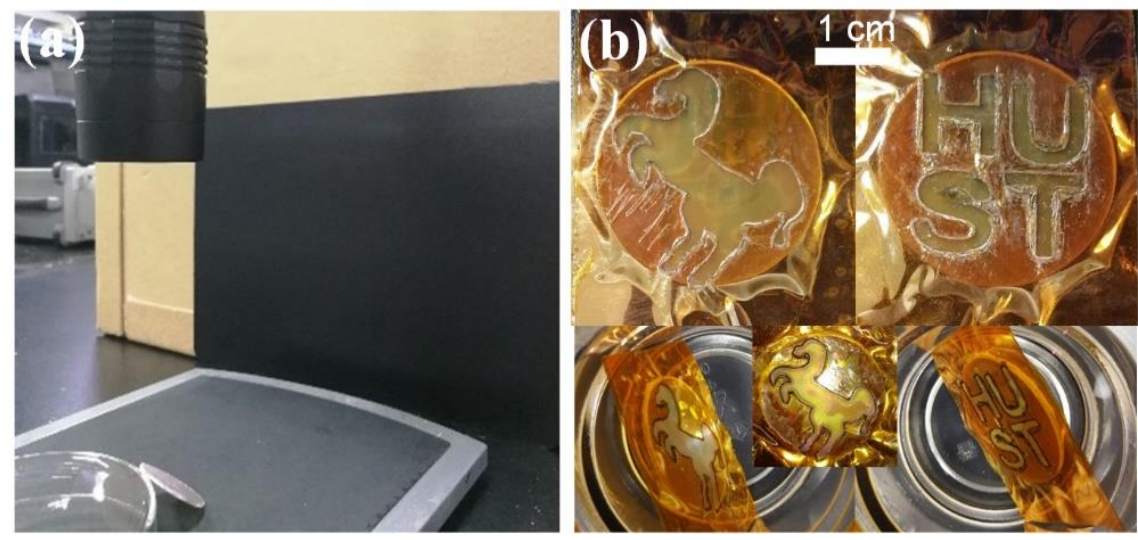

Figure S5. (a) Illustration for experimental setup to project and record the reflected color of bulk PC devices; (b) An example shows the process of stencil screen printing.

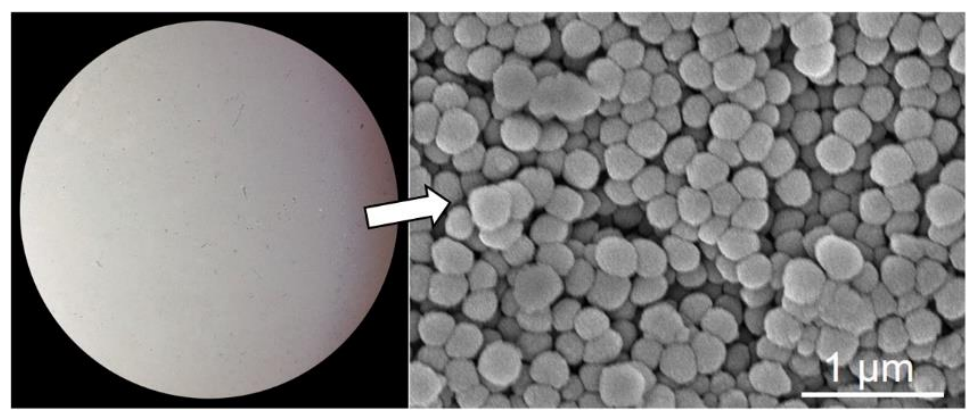

Figure S6. Image of the bulk PC with 7 wt \% DVB under the long-time etching (30 min; Mixed solvent, $\left.V_{\text {Ethyl Acetate }}: \mathrm{V}_{\text {Anhydrous Ethanol }}=1: 1\right)$ and its SEM image.

\section{References}

(1) Nucara, L.; Greco, F.; Mattoli, V. Electrically Responsive Photonic Crystals: a Review. J. Mater. Chem. C 2015, 3, 8449-8467.

(2) Kanai, T.; Nakashima, S.; Oki, T. Protection Against a Wide UV Wavelength Range by Bragg Reflection from Polycrystalline Colloidal Photonic Crystals. J. Mater. 
Chem. C 2019, 7, 7512-7515.

(3) Kawamura, A.; Kohri, M.; Morimoto, G.; Nannichi, Y.; Taniguchi, T.; Kishikawa, K. Full-Color Biomimetic Photonic Materials with Iridescent and Non-Iridescent Structural Colors. Sci. Rep. 2016, 6, 33984. 